\title{
8 Grand summary and conclusion
}

This monograph makes two major contributions to the investigation of the V2phenomenon which build on the well-established insight that the V2-order is a derived word order establishing a dependency between the base position and the surface position of the finite verb. First, the investigation provides empirical evidence from linguistic and psycholinguistic investigations for the hypothesis that the lexical content of the V2-verb is reconstructed into and only interpreted in its base position. Verb reconstruction, therefore, provides a case in which language comprehension closely follows grammatical principles. Second, the discussion refines some details of the reconstruction process and relates it to other general properties of language processing in German.

In the following, I briefly summarize the chapters and recapitulate the main conclusions and open issues.

Part I set the scene with an introduction into the broader phenomenon of V2movement and the presentation of the V2-Reconstruction Hypothesis - the core idea on which this publication is based.

In Chapter 2, I illustrated the main properties of the V2-phenomenon. First, there must be a movement dependency between the base position of the finite verb and the V2-position with the latter being a derived position because, under certain conditions, specific verbs may only appear in the base position but not in the V2-position, whereas the reverse pattern is unattested. Second, V2-movement applies exceptionless, i. e. it cannot be suspended. In some cases, the application of V2-movement conflicts with another rule of grammar and would result in an ungrammatical structure. Crucially, however, the non-application of V2-movement in such cases also leads to ungrammaticality. The only possibility is to alter the sentence, e. g. by using a different verb form, such that both grammatical requirements can be satisfied. Third, V2-movement targets one specific position in the left periphery of the clause. While the filling of the prefield shows larger variation giving rise to V1- or V3-pattern, these patterns are very restricted in their interpretation and use conditions.

Chapter 3 introduced the central assumption, namely the V2-Reconstruction Hypothesis, according to which V2-movement affects only the finiteness features while the lexical part of the verb is pied-piped. Consequently, the lexical part has to be reconstructed into its base position, which is is also the only locus at which the lexical content of the verb is interpreted. Two groups of evidence were presented in support of the V2-Reconstruction Hypothesis: First, I showed that the V2-position is systematically filled with surface elements that are insufficient for 
interpretation (particle verbs), semantically empty (periphrastic tun), or outright ungrammatical if interpreted at this point (verb doubling). Second, using semantic operators which require a c-command relation, such as association with focus, verbal NPIs and sentence negation, I provided evidence that the finite verb must be interpreted in its base position. Taken together, the results strongly support the V2-Reconstruction Hypothesis.

All in all, Part I provided the empirical and theoretical background to understand the significance and the implications of the V2-Reconstruction Hypothesis. Additionally, it also provided an empirical basis for the validity of the V2-Reconstruction Hypothesis whose predictions concerning language processing were applied in the next part. The discussion of Part I has shown that V2-movement is a rather abstract mechanism that attracts only the finiteness features or, as the data from complementizer agreement indicates, only the subset of subject-agreement features. However, it still remains unclear, why these features have to be realized in the left periphery in V2-languages. There exist several sophisticated accounts that connect V2-movement with the illocutionary potential of main clauses. As mentioned in Chapter 2, for now, these accounts seem to be language-specific. They may not be generalizable for all V2-languages and, moreover, do not explain why such a feature movement seems to be unnecessary in the majority of languages.

Part II provided a general introduction to language processing and sentence processing in German, followed by a series of on-line experiments which tested the predictions of the V2-Reconstruction Hypothesis. Finally, I explored some specifics of the reconstruction process based on theoretical arguments.

In Chapter 4, I sketched the basic properties of an on-line language comprehension model with a focus on the syntactic domain. I illustrated that deviations from strict on-line processing, as assumed by the V2-Reconstruction Hypothesis, occur regularly and are handled by one of two different processing strategies: anticipatory analysis with potential subsequent repairs, or delayed processing. Comparison with well-established manifestations of these two strategies in other languages and domains, especially the absence of garden paths effects, indicated that V2-clauses adhere to delayed processing. Additionally, a delayed processing as assumed under the V2-Reconstruction Hypothesis is also preferable for reasons of economy because the V2-position is frequently occupied by verbs that are either uninformative (auxiliaries, modals, etc.) or misleading with respect to the meaning of the predicate (particle verbs).

Chapter 5 reviewed previous findings regarding the processing of the core argument structure in German sentences. The review showed that argument orders that deviate from the canonical NOM $<$ ACC $<$ DAT order give rise to immediate pro- 
cessing responses, which are more local for scrambling and long-lasting for topicalization. These findings provided the background for experimental investigations of the influence of lexical V2-verbs on the processing of arguments. In this light, I reviewed on-line processing experiments on thematic structure, attachment ambiguities and scope computing in German. The results indicated that potentially verb-related effects only appeared rather late in the clauses as predicted by the V2-Reconstruction Hypothesis.

In Chapter 6, I presented four self-paced reading experiments that tested predictions of the V2-Reconstruction Hypothesis concerning on-line sentence processing. The experiments on verbal NPIs and the one on infinitive selection showed that the finite verb is automatically reconstructed into the first potential base position. Only subsequent interpretation could reveal whether insertion of the verb led to ungrammaticality, e.g. in case of non-licensed NPIs or mismatching infinitive forms, which was reflected by increased reading times. The experiment on the interaction of verb types and argument order showed a verbspecific modulation of the argument-order preference at the reconstruction site of the verb. Additionally, the experimental manipulation led to other, less clear-cut, correlates appearing later and also earlier in the clause which must be further investigated to allow a clear interpretation.

In Chapter 7, I examined three aspects of how the reconstruction process should look like. First, subject-initial V2-clauses are special in that their structural representation can be generated with fewer restructuring operations. Second, I contrasted two realizations of verb reconstruction: lowering the verb cascadically after every incoming unit, or inserting incoming units directly before the verb. By applying the logic of temporary constituency to Right Node Raising structures in German V2-clauses, I constructed an argument against the cascading analysis and in favor of the insertion approach. Third, I argued that only the subject-verb agreement features on the finite verb are immediately interpreted. Other aspects of the verb are only interpreted after every incoming argument. The interpretation may be extended or altered during the course of the processing. However, once unambiguous cues for the clause-final base position are encountered, the parse is completed and the core meaning cannot be altered again, as evidenced by restrictions on extraposition.

Based on explicit assumptions about a human processing device, I explicated in this part how the predictions of the V2-Reconstruction Hypothesis are reflected in previous research on sentence comprehension in German. Furthermore, I presented the results of four specifically designed studies as additional support of the V2-Reconstruction Hypothesis. Finally, I discussed some details of the structural analysis and its relation to semantic interpretation in on-line processing. The experimental investigation of the V2-Reconstruction Hypothesis is the major contri- 
bution of this work. The results support the hypothesis that there is an asymmetry between the syntactic realization of the finite verb and the semantic interpretation. While I have shown in Part I that this asymmetry is operative in the realm of grammar, the findings of this part showed that this asymmetry also holds on the level of language processing. This may provide the strongest case of grammar overruling inherent principles of on-line processing. It is clear that the investigations here can only be regarded as the beginning of an experimental endeavor aiming in a detailed understanding of how the linguistic representations are constructed in V2-environments. After all, the experimental results presented here indicate that at least some meaning components of the verb, such as polarity-sensitivity and infinitive selection, are activated at a position different from the surface occurrence. This is an important result for any research on sentence processing in V2-languages because until now it has mostly been assumed, implicitly or explicitly, and without positive evidence that V2-sentences are processed/interpreted strictly incrementally. While future research must show which aspects enter the sentence processing routine immediately, the findings of this study indicate that some information is only evaluated at the reconstruction site of the verb.

Taken together, this investigation presented evidence which shows that German V2-order is derived from an underlying V-final order. Moreover, I presented a hypothesis according to which the underlying base position is not only of relevance for grammar models but it is also the locus where the lexical content of the V2moved verb will be interpreted. In support of this hypothesis, I have offered empirical evidence from grammatical regularities and from on-line processing experiments, confirming the psychological reality of the hypothesis.

Finally, I would like to add two remarks, one about the nature of V2-movement and one about the generality of the findings: First, the findings indicate that V2movement is the consequence of the requirement to realize subject-verb agreement features in the left periphery. It remains open why these features have to be realized in the V2-position in V2-languages only. However, whereas the findings reveal that the lexical content of the V2-verb only enters the processing at a later point, it has been noted that the realization of the agreement features immediately impacts language processing. This shows that V2-movement is best characterized as I-to-C movement, which also becomes evident in languages that realize agreement features exclusively by clitics (Warpiri) or auxiliaries (O'odham) - these languages never move lexical verbs. Having this in mind, I must clearly oppose to the claim that V2-movement is non-syntactical in nature, as e. g. argued in Zwart (2017). As stated above, the pied-piping of other material, such as the lexical verb, is due to extrasyntactic factors whereas the movement of the agreement features is purely syntactic. In this way, deeming V2-movement as a whole non-syntactic 
misses crucial details of its nature. Second, the argumentation of this contribution was mainly based on German. At numerous points, however, I drew comparisons to other V2-languages. Generally, I assume that the cause of V2-movement is identical in all V2-languages, namely that subject-verb agreement features must be realized in the left periphery. Consequently, the V2-Reconstruction Hypothesis applies to all other V2-languages as well. However, VO-languages, such as the Scandinavian languages, differ not only in the locus of the base position of the finite verb but, as I mentioned above, VO-parsing assumably differs parametrically from OV-parsing. This must certainly be considered if the findings of this investigation will be adapted to those languages. 
